\title{
On the equivalence of two expected average cost criteria for semi- Markov control processes
}

\author{
Anna Jaśkiewicz ${ }^{1}$ \\ Institute of Mathematics, Wrocław University of Technology, Wybrzeże Wyspiańskiego \\ 27, 50-370 Wrocław, Poland (e-mail: ajaskiew@im.pwr.wroc.pl)
}

\begin{abstract}
The two expected average costs used in the theory of semi-Markov control processes with a Borel state space are considered. Under some stochastic stability conditions, we prove that the two criteria are equivalent in the sense that they lead to the same optimality equation.
\end{abstract}

Key Words: Semi-Markov control models, Borel state space, average cost optimality equation.

\section{The model}

In this paper we study two basic optimality criteria used in the theory of semi-Markov control processes (see $[4,12,19]$, for instance). According to the first one, the average cost is the lim sup of the expected total costs over a finite number of jumps divided by the expected cumulative time of these jumps. According to the second definition, the average cost is the limsup of the expected total costs over the finite deterministic horizon divided by the length of the horizon. We shall call them (following Feinberg [4]) the ratio-average cost and time-average cost, respectively.

Some partial results on the equivalence of the two criteria were given in the books by Ross [19] and Puterman [18], but only for countable state space models and stationary policies. A fairly complete treatment of the problem for semi-Markov control models with countably many states can be found in [20]. The main objective in this paper is to prove the equivalence of the mentioned criteria in a Borel state space framework. A more detailed presentation of the perspective in which our research is done is given at the end of this section.

We shall use the following terminology. A Borel subset, say $S$, of a complete, separable metric space is called a Borel space, and it is equipped with the Borel $\sigma$-algebra, denoted by $\mathcal{B}(S)$.

A semi-Markov control model operates as follows. The process is observed at time $t=0$ to be in some state $x_{0}=x \in X$, where $X$ is Borel state space. At that time an action $a_{0}=a \in A(x)$ is chosen, where $A(x)$ is a compact set of actions available in state $x$. The set of all actions is $A$ and is also assumed to be a Borel state space. By

$$
K:=\{(x, a): x \in X, a \in A(x)\}
$$

we define the admissible pair set as a Borel subset of $X \times A$.

\footnotetext{
${ }^{1}$ Research supported by KBN Grant 5 P03A 01420
} 
If the current state is $x$ and action $a$ is selected, then the immediate cost of $c_{1}(x, a)$ is incurred and the system remains in state $x_{0}=x$ for a random time $T$ with the cumulative distribution depending only on $x$ and $a$. The cost of $c_{2}(x, a)$ per unit time is incurred until the next transition occurs. Afterwards the system jumps to the state $x_{1}=y$ according to the probability measure (transition law) $q(\cdot \mid x, a)$. This procedure yields a trajectory $\left(x_{0}, a_{0}, t_{1}, x_{1}, a_{1}, t_{2}, \ldots\right)$ of some stochastic process, where $x_{n}$ is the state, $a_{n}$ is control variable and $t_{n}$ is the time of the $n$th transition, $n=0,1, \ldots$

REMARK 1: In this work we shall slightly abuse the notation. Namely, the state and action variables will be denoted by small letters $x_{n}$ and $a_{n}$ as their values. Other random variables defined on the space of all trajectories will be written by means of the capital letters, e.g. $T_{n}$ - the random time of $n$th transition, for $n=1,2, \ldots$ with $T_{0}:=0$.

Let $H_{n}$ be the space of admissible histories up to the $n$th transition:

$$
H_{n}:=(K \times[0,+\infty))^{n} \times X \quad \text { where } \quad H_{0}=X .
$$

An element $h_{n}$ of $H_{n}$ is called a partial history of the process and is of the form

$$
h_{n}:=\left(x_{0}, a_{0}, t_{1}, \ldots, x_{n-1}, a_{n-1}, t_{n}, x_{n}\right) .
$$

A control policy (or policy) is a sequence $\pi=\left\{\pi_{n}\right\}$, where each $\pi_{n}$ is a conditional probability $\pi_{n}\left(\cdot \mid h_{n}\right)$ on the control set $A\left(x_{n}\right)$, given the entire history $h_{n}$ such that

$$
\pi_{n}\left(A\left(x_{n}\right) \mid h_{n}\right)=1 \quad \forall h_{n} \in H_{n}, \quad n=0,1, \ldots
$$

The class of all policies is denoted by $\Pi$.

Let $F$ be the set of all Borel measurable mappings $f: X \mapsto A$ such that $f(x) \in A(x)$ for each $x \in X$. It is well known that $F$ is nonempty if the sets $A(x)$ are compact (Corollary 1 in [3]). A sequence $\pi=\left\{\pi_{n}\right\}, n=0,1, \ldots$ is a (nonrandomized) stationary policy iff there is some $f \in F$ such that $\pi_{n}\left(\cdot \mid h_{n}\right)$ is concentrated at $f\left(x_{n}\right) \in A\left(x_{n}\right)$ for all $h_{n} \in H_{n}$ and $n=0,1, \ldots$ Thus any stationary policy $\pi=(f, f, \ldots)$ can be identified with $f \in F$.

Let $(\Omega, \mathcal{F})$ be the measurable space consisting of the sample (or trajectory) space $\Omega:=(X \times A \times[0,+\infty))^{\infty}$ and the corresponding product $\sigma$-algebra $\mathcal{F}$. According to the theorem of C. Ionescu Tulcea (Proposition V.1.1 in [13] or Chapter 7 in [1]), for each initial state $x_{0}=x \in X$ and each policy $\pi \in \Pi$, there exists a unique probability measure $P_{x}^{\pi}$ on $\mathcal{F}$ such that for all $B \in \mathcal{B}(A), D \in \mathcal{B}(X)$ and $h_{n}=\left(x_{0}, a_{0}, t_{1}, \ldots, x_{n-1}, a_{n-1}, t_{n}, x_{n}\right)$ in $H_{n}, n=1,2, \ldots$,

$$
\begin{gathered}
P_{x}^{\pi}\left(x_{0}=x\right)=1, \\
P_{x}^{\pi}\left(a_{n} \in B \mid h_{n}\right)=\pi_{n}\left(B \mid h_{n}\right), \\
P_{x}^{\pi}\left(x_{n+1} \in D \mid h_{n}, a_{n}, t_{n+1}\right)=q\left(D \mid x_{n}, a_{n}\right) .
\end{gathered}
$$

Moreover,

$$
P_{x}^{\pi}\left(T_{n+1}-T_{n} \leq t \mid h_{n}, a_{n}\right)=P_{x_{n}}^{a_{n}}\left(T_{n+1}-T_{n} \leq t\right)
$$


We remind that the last equality is a consequence of our assumption that the conditional distribution of the difference $T_{n+1}-T_{n}$ given the whole history up to the $n$th pair $\left(x_{n}, a_{n}\right) \in$ $K$ depends only on $x_{n}$ and $a_{n}$. As usual, by $E_{x}^{\pi}$ we shall denote the expectation operator with respect to the probability measure $P_{x}^{\pi}$.

Further, set $\tau(x, a)$ for the mean holding (sojourn) time, i.e.

$$
\tau(x, a):=\int_{0}^{\infty} t P_{x}^{a}(d t)=E_{x}^{a} T
$$

Recall that $T$ is the random time spent in state $x$.

Let $\pi \in \Pi, x \in X$ and $t \geq 0$ be fixed. Put

$$
N(t):=\max \left\{n \geq 0: T_{n} \leq t\right\}
$$

as the counting process. Later on, we shall make some assumptions under which $P_{x}^{\pi}(N(t)<$ $\infty)=1$ (see Remark 2). Now we can define the two kind of expected average costs as

$$
J(x, \pi):=\limsup _{n \rightarrow \infty} \frac{E_{x}^{\pi}\left(\sum_{k=0}^{n-1} c\left(x_{k}, a_{k}\right)\right)}{E_{x}^{\pi} T_{n}},
$$

and

$$
j(x, \pi):=\limsup _{n \rightarrow \infty} \frac{E_{x}^{\pi}\left(\sum_{k=0}^{N(t)} c\left(x_{k}, a_{k}\right)\right)}{t},
$$

where

$$
c(x, a):=c_{1}(x, a)+\tau(x, a) c_{2}(x, a) .
$$

We will need the following assumptions:

B Basic assumptions:

(i) for each $x \in X$, the set $A(x)$ is compact metric space;

(ii) for each $x \in X, c(x, \cdot)$ is lower semicontinuous on $A(x)$;

(iii) for each $x \in X$ and every Borel set $D \subset X$, the function $q(D \mid x, \cdot)$ is continuous on $A(x)$;

(iv) for each $x \in X, \tau(x, \cdot)$ is continuous on $A(x)$, and there exist positive constants $b$ and $B$ such that

$$
b \leq \tau(x, a) \leq B
$$

for all $(x, a) \in K$;

(v) there exist a constant $L>0$ and a Borel measurable function $V: X \mapsto[1, \infty)$ such that $|c(x, a)| \leq L V(x)$ for every $(x, a) \in K$;

(vi) for each $x \in X$, the function

$$
\int_{X} V(y) q(d y \mid x, \cdot)
$$


is continuous on $A(x)$.

GE Geometric ergodicity assumptions:

(i) there exists a Borel set $C \subset X$ such that for some $\lambda \in(0,1)$ and $\eta>0$, we have

$$
\int_{X} V(y) q(d y \mid x, a) \leq \lambda V(x)+\eta 1_{C}(x)
$$

for each $(x, a) \in K ; V$ is the function introduced in $(\mathbf{B}, \mathrm{v})$;

(ii) the function $V$ is bounded on $C$, i.e.

$$
v_{C}:=\sup _{x \in C} V(x)<\infty
$$

(iii) there exist some $\delta \in(0,1)$ and a probability measure $\mu$ concentrated on the Borel set $C$ with the property that

$$
q(D \mid x, a) \geq \delta \mu(D)
$$

for each Borel set $D \subset C, x \in C$ and $a \in A(x)$.

For any Borel measurable function $u: X \mapsto R$ we define the weighted norm as

$$
\|u\|_{V}:=\sup _{x \in X} \frac{|u(x)|}{V(x)}
$$

By $L_{V}^{\infty}$ we denote the Banach space of all Borel measurable functions $u$ for which $\|u\|_{V}$ is finite.

We also make two additional assumptions on the sojourn time $T$ :

R Regularity condition:

there exist $\epsilon>0$ and $\beta<1$ such that

$$
P_{x}^{a}(T \leq \epsilon) \leq \beta
$$

for all $x \in C$ and $a \in A(x)$.

I Uniform integrability condition:

$$
\lim _{t \rightarrow \infty} \sup _{x \in C} \sup _{a \in A(x)} P_{x}^{a}(T>t)=0
$$

Assumption $(\mathbf{G E})$ is basic for this paper. Inequality (GE, i) is called the "drift inequality" and the set $C$ satisfying (GE, iii) is called "small set" [10]. They imply that the embedded state process $\left\{x_{n}\right\}$ governed by a stationary policy is a positive recurrent aperiodic Markov chain with the unique invariant probability measure, denoted by $\pi_{f}$ 
(consult Theorem 11.3.4 and page 116 in [10]). Moreover, $\left\{x_{n}\right\}$ is $V$-uniformly ergodic (Theorem 2.3 in [11]), that is, there exist $\theta>0$ and $\alpha \in(0,1)$ such that

$$
\left|\int_{X} u(y) q^{n}(d y \mid x, f)-\int_{X} u(y) \pi_{f}(d y)\right| \leq V(x)\|u\|_{V} \theta \alpha^{n}
$$

for every $u \in L_{V}^{\infty}$ and $x \in X, n \geq 1$. Here $q^{n}(\cdot \mid x, f)$ denotes the $n$-stage transition probability induced by $q$ and a stationary policy $f$. Condition $(\mathbf{G E})$ is often used in the theory of control processes and stochastic games on Borel state spaces [5, 17].

Assumptions ( R, I) are not imposed on the processs, when we work with the ratioaverage criterion (1). However, if we do wish do deal with the time-average cost (2), condition $(\mathbf{R})$ is needed. It ensures that the infinite number of transitions does not occur in a finite interval [19]. Note also that conditions $(\mathbf{R}),(\mathbf{I})$ do not coincide with assumption (B, iv). ( $\mathbf{R})$ implies only that $\tau(x, a)>\epsilon(1-\beta)$ for $x \in C$. The reader who is interested in examples is referred to $[19,21]$.

The literature that deals with semi-Markov control processes under ratio-average cost is very wide, see e.g. $[6,12,18,19,20,21]$ and references therein. There are only few papers examining the time-average cost $[4,12,18,19,20,23]$. The reason lies in the fact that it is somewhat easier to study the ratio-average cost. Generally, these two criteria may have nothing to do with each other. In other words, they may lead to different cost and optimal policies. Such situations happen even if the state space is countable and there are no ergodic (recurrence) properties of the transition probabilities induced by stationary policies. Ross [19] first observed that the two expected costs coincide for stationary policies if the embedded Markov chain satisfies some strong recurrence condition. Much stronger result was given by Schäl [20], who assumed certain stochastic stability assumptions and proved that the optimal expected costs are same in both approaches mentioned above. Moreover, Schäl showed that the average optimality equation is the same in both cases and has a solution. We point out that Schäl's paper was devoted to semi-Markov control processes with countable state space. The optimality equations for semi-Markov decision models with ratio-average criterion (1) and Borel state spaces were derived quite recently $[6,7]$. In this work, our goal is to generalize the result of Ross for (uncountable) Borel state space. Under the $V$-geometric ergodicity assumption [10], we shall prove that criteria (1) and (2) coincide when the process is induced by stationary policies. At the same time we show that the optimality equation, established in $[6,7,24]$ for the models with the cost (1) remains valid for the time-average cost (2).

As in earlier papers $[19,20]$, some parts of our proof employs basic facts from renewal theory. Certain consequences of $V$-geometric ergodicity given in $[10,11]$ enable us to apply the optional sampling theorem of Doob [14], which is the core of the proof.

For convenience of the reader we recall the optimality equation, which is our point of departure. The proofs are given in $[6,7,24]$. They are based on different methods.

Proposition: Let $(\mathbf{B}, \mathbf{G E})$ hold. Then there exist a function $h \in L_{V}^{\infty}$ and a constant $g$ 
such that

$$
h(x)=\min _{a \in A(x)}\left[c(x, a)+\int_{X} h(y) q(d y \mid x, a)-g \tau(x, a)\right]
$$

for all $x \in X$. Moreover,

$$
g=\inf _{\pi \in \Pi} J(x, \pi)=J\left(x, f^{*}\right),
$$

where $f^{*} \in F$ is a Borel measurable selector of minima on the right side of (3), for each $x \in X$.

\section{Main result}

We begin with presenting our main result in this paper:

Theorem: Assume (B,GE,R,I). Then

(a)

$$
g=\inf _{\pi \in \Pi} j(x, \pi)
$$

$$
j(x, f)=J(x, f) \quad \text { for each } \quad f \in F .
$$

Corollary: Any $f^{*} \in F$ as in Proposition is average optimal with respect to timeaverage criterion (2).

The above results are new. As already noted, some precedessors of our theorem were established in [19, 20], but only for semi-Markov control models with countable state spaces.

Our proof owes much to Ross [19] and Schäl [20], especially in the parts connected with renewal theory. In order to apply the optional sampling theorem, we have to study some consequences of the stochastic stability ( $V$-geometric ergodicity) assumptions and certain measurability issues.

1. Some corollaries of "drift inequality." For the set $C$ we define the first return time on $C$ as

$$
N_{C}:=\min \left\{n \geq 1: x_{n} \in C\right\} .
$$

If we do wish to distinguish different return times, we write $N_{C}(k)$ for the random time of $k$ th visit to $C$ : these are defined inductively by

$$
N_{C}(1):=N_{C}
$$


and

$$
N_{C}(k):=\min \left\{n>N_{C}(k-1): x_{n} \in C\right\} .
$$

Lemma 1: Let (GE, i) hold and let $\left\{x_{n}\right\}$ denote the state space process under arbitrarily fixed policy $\pi \in \Pi$. Then for each initial state $x \in X$, any function $u \in L_{V}^{\infty}$ and $n \geq 1$ we obtain

(a)

$$
E_{x}^{\pi}\left(\left|u\left(x_{n}\right)\right| ; N_{C} \geq n\right) \leq\|u\|_{V} \lambda^{n-1}\left(\lambda V(x)+\eta 1_{C}(x)\right),
$$

$$
\limsup _{n \rightarrow \infty} E_{x}^{\pi}\left(\left|u\left(x_{n}\right)\right| ; N_{C} \geq n\right)=0
$$

$$
E_{x}^{\pi}\left|u\left(x_{n}\right)\right| \leq\|u\|_{V} \lambda^{n} V(x)+\eta\left(1+\ldots+\lambda^{n-1}\right) .
$$

Proof: Parts (a) and (c) can be obtained by iteration of (GE, i) on the set $X \backslash C$, and $X$, respectively. Part (b) follows from (a).

Our next lemma has a well-known counterpart in the theory of Markov chains called Comparison Theorem [10]. The proof is based on Dynkin's Formula and proceeds along the same lines as in [10], pages 263-264.

Lemma 2: Suppose that nonnegative functions $V, r, s$ satisfy the relationship

$$
\int_{X} V(y) q(d y \mid x, a) \leq V(x)-r(x)+s(x) .
$$

Then for each $x \in X, \pi \in \Pi$ and any stopping time $\mathcal{S}$, we have

$$
E_{x}^{\pi}\left[\sum_{k=0}^{\mathcal{S}-1} r\left(x_{k}\right)\right] \leq V(x)+E_{x}^{\pi}\left[\sum_{k=0}^{\mathcal{S}-1} s\left(x_{k}\right)\right]
$$

LEMma 3: If assumption (GE, i) holds, then the following bounds are satisfied for all $x \in X:$

(a)

$$
E_{x}^{\pi}\left(\sum_{n=0}^{N_{C}-1} V\left(x_{n}\right)\right) \leq \psi(x):=\frac{1}{1-\lambda} V(x)+\frac{\eta}{1-\lambda} 1_{C}(x)
$$

$$
E_{x}^{\pi} N_{C} \leq \phi(x):=\frac{1}{\ln (1 / \lambda)}\left(\ln V(x)+\frac{\eta}{\lambda} 1_{C}(x)\right) .
$$


Proof: (a) It follows from Lemma 2 for the stopping time $N_{C}$ and the functions $r:=$ $(1-\lambda) V, s:=\eta 1_{C}$.

(b) From Jensen's inequality and (GE, i), it follows that

$$
\int_{X} \ln V(y) q(d y \mid x, a) \leq \ln V(x)+\ln \lambda+\frac{\eta}{\lambda} 1_{C}(x)
$$

and the rest is obtained by applying Lemma 2 .

2. An analysis of returns to the set $C$. For any $\pi \in \Pi, t \geq 0$ and $x \in X$, let

$$
M_{x}^{\pi}(t):=E_{x}^{\pi}\left(\sum_{m=1}^{\infty} 1_{\left[x_{m} \in C, T_{m} \leq t\right]}\right)=\sum_{m=1}^{\infty} P_{x}^{\pi}\left(x_{m} \in C, T_{m} \leq t\right)
$$

be the expected number of visits in the small set $C$ during the time interval $[0, t]$.

Define the distribution function $H$ in the following way:

$$
H(t):= \begin{cases}\beta, & t \in[0, \epsilon) \\ 1, & t \geq \epsilon,\end{cases}
$$

where $\beta$ and $\epsilon$ are taken from assumption $(\mathbf{R})$. For $t<0$ set $H(t):=0$. The corresponding renewal function is given by

$$
M(t):=\sum_{n=0}^{\infty} H^{n *}(t)
$$

where $H^{n *}$ is the $n$-fold convolution of $H\left(H^{0 *}:=1\right.$ on $[0,+\infty]$ and $H^{0 *}:=0$ for $\left.t<0\right)$. By $(\mathbf{R})$, we have

$$
P_{x}^{\pi}\left(T_{N_{C}} \leq t\right) \leq P_{x}^{\pi}\left(T_{1} \leq t\right) \leq H(t),
$$

for each $x \in C$. Making use of this fact and using standard methods from renewal theory, one can show the following facts.

Lemma 4: For $\pi \in \Pi, x \in X$ and $t, h \geq 0$, we have:

(a) $M_{x}^{\pi}(t) \leq M(t)<\infty$,

(b) $M_{x}^{\pi}(t+h)-M_{x}^{\pi}(t) \leq M(h)$,

(c) if additionally $z(t)$ is a bounded, nonincreasing, and nonnegative function such that $\lim _{t \rightarrow \infty} z(t)=0$, then

$$
\lim _{t \rightarrow \infty} \frac{1}{t} \int_{0}^{t} z(t-u) M_{x}^{\pi}(d u)=0 .
$$

Proof: (a) This part follows from [19], and the fact that $\left\{T_{n+1}-T_{n}\right\}, n=0,1, \ldots$ are conditionally independent random variables given the history of the states and actions process. 
(b) The proof is similar to that of part (a); see also [2].

(c) Use (a) and (b) and the fact that $\frac{M(t)}{t} \rightarrow \frac{1}{\epsilon(1-\beta)}$, when $t \rightarrow \infty$ (see Key Renewal Theory in [19]).

3. The proof of Theorem. We start with some helpful notation. For any $n \geq 1$, we write $\mathcal{F}_{n}$ for the $\sigma$-algebra of all events up to the $n$th state. Let $\xi$ be a stopping time relative to $\left\{\mathcal{F}_{n}\right\}$. By $\mathcal{F}_{\xi}$, we denote the $\sigma$-algebra of all events up to the stopping time $\xi$, i.e., $\mathcal{F}_{\xi}:=\left\{D \in \mathcal{F}:[\xi=n] \cap D \in \mathcal{F}_{n}, \forall n \geq 0\right\}$.

In this section we accept all our assumptions. For clear-sighted analysis, we divide the proof into a sequence of Lemmas.

Let $h_{n}=\left(x_{0}, a_{0}, t_{1}, \ldots, a_{n-1}, t_{n}, x_{n}\right) \in H_{n}$. We put $h_{n}^{\prime}:=\left(x_{0}, a_{0}, t_{1}, \ldots, a_{n-1}, t_{n}\right)$. For any policy $\pi \in \Pi$ and $m \geq 1$, the conditional policy is formally defined by setting

$$
\pi_{n}\left[h_{m}^{\prime}\right]\left(\cdot \mid h_{n}\right):=\pi_{n+m}\left(\cdot \mid h_{m}^{\prime}, h_{n}\right) .
$$

By $E_{x_{m}}^{\pi\left[h_{m}^{\prime}\right]}$, we denote the (conditional) expectation operator corresponding to the conditional probability measure induced by $\pi\left[h_{m}^{\prime}\right]$, the transition law $q$ and the holding time distribution. In a similar way, we define $\pi\left[x_{0}, a_{0}\right], E_{x_{0}}^{\pi\left[x_{0}, a_{0}\right]}$ etc.

Let $h_{n}^{\prime}$ be fixed. Put $\tilde{h}_{n}:=x_{n}$ and $\tilde{h}_{m}:=\left(x_{n}, a_{n}, t_{n+1}, \ldots, t_{m}, x_{m}\right)$ for $m>n$. Identify $\left(h_{n}^{\prime}, \tilde{h}_{m}\right)$ with $h_{m}$. With any conditional policy $\pi\left[h_{m}^{\prime}\right]$ we associate the usual policy $\hat{\pi}=$ $\left\{\hat{\pi}_{m}\right\}$, where

$$
\hat{\pi}_{m}\left(\cdot \mid \tilde{h}_{m}\right)=\pi_{m}\left(\cdot \mid\left(h_{n}^{\prime}, \tilde{h}_{m}\right)\right)=\pi_{m}\left(\cdot \mid h_{m}\right) .
$$

Here $x_{n}$ is treated as the initial state. Below we present a simple auxiliary result.

Lemmma 5: Let $u: \Omega \mapsto R$ be a Borel measurable function such that $E_{x}^{\pi}|u|<\infty, x \in X$, $\pi \in \Pi$. Then

(a)

$$
u^{*}(x):=\sup _{\pi \in \Pi} E_{x}^{\pi} u\left(x_{0}, a_{0}, T_{1}, \ldots\right)
$$

is universally measurable function,

$$
E_{x_{n}}^{\pi\left[h_{n}^{\prime}\right]} u\left(x_{n}, a_{n}, T_{n+1}, \ldots\right)=E_{x_{n}}^{\hat{\pi}} u\left(x_{n}, a_{n}, T_{n+1}, \ldots\right) \leq \sup _{\pi \in \Pi} E_{x_{n}}^{\pi} u\left(x_{n}, a_{n}, T_{n+1}, \ldots\right) .
$$

Proof: For part (a) see [1, 22]. Part (b) is obvious.

Lemma 6: For any $\pi \in \Pi, x \in X$ and $t \geq 0$, we have:

$$
E_{x}^{\pi}\left(\sum_{n=0}^{N(t)}\left|c\left(x_{n}, a_{n}\right)\right|\right) \leq L \psi(x)+L \psi_{C} M(t)<\infty
$$


(b)

$$
E_{x}^{\pi}\left(\sum_{n=0}^{N(t)} \tau\left(x_{n}, a_{n}\right)\right) \leq B \phi(x)+B \phi_{C} M(t)<\infty,
$$

with $\psi$ and $\phi$ as defined in Lemma 3 and $\psi_{C}:=\sup _{x \in C} \psi(x), \phi_{C}:=\sup _{x \in C} \phi(x)$ (see $(\mathbf{G E}, \mathrm{ii}))$. The constants $L, B$ are from $(\mathbf{B}, \mathrm{iv}, \mathrm{v})$.

Proof: (a)

$$
\begin{aligned}
E_{x}^{\pi} & \left(\sum_{n=0}^{N(t)}\left|c\left(x_{n}, a_{n}\right)\right|\right)=E_{x}^{\pi}\left(\sum_{n=0}^{N_{C}-1 \wedge N(t)}\left|c\left(x_{n}, a_{n}\right)\right|\right) \\
& +\sum_{m=1}^{\infty} E_{x}^{\pi}\left(E_{x}^{\pi}\left[\sum_{n=N_{C}(m)}^{N_{C}(m+1)-1 \wedge N(t)}\left|c\left(x_{n}, a_{n}\right)\right| \mid \mathcal{F}_{N_{C}(m)}\right]\right)= \\
& E_{x}^{\pi}\left(\sum_{n=0}^{N_{C}-1 \wedge N(t)}\left|c\left(x_{n}, a_{n}\right)\right|\right)+ \\
& \left.\sum_{m=1}^{\infty} E_{x}^{\pi}\left(E_{x_{m}}^{\pi\left[h_{m}^{\prime}\right]}\left[\sum_{n=0}^{N_{C}-1 \wedge N\left(t-T_{m}\right)} \mid c\left(x_{n}, a_{n}\right)\right]\right] ; x_{m} \in C, T_{m} \leq t\right) \leq \\
& L \psi(x)+L \psi_{C} M(t)<\infty,
\end{aligned}
$$

The second equality is due to the strong Markov property generalized to arbitrary policies (see [20] for a similar argument). The conclusion follows from Lemmas 3(a), 4(a) and $5(\mathrm{~b})$. The proof of part (b) is similar to that of part (a).

REMARK 2: If we replace $|c(x, a)|$ by $|c(x, a)|+1$ in the proof of Lemma 6(a), we obtain

$$
E_{x}^{\pi}\left(\sum_{n=0}^{N(t)}\left(1+\left|c\left(x_{n}, a_{n}\right)\right|\right)\right) \leq(L+1) \psi(x)+(L+1) \psi_{C} M(t)<\infty .
$$

Hence, it follows that $E_{x}^{\pi} N(t)<\infty$ and consequently $N(t)<\infty P_{x}^{\pi}$-a.e.

Lemma 7: For any $\pi \in \Pi, x \in X$, we have:

$$
\lim _{t \rightarrow \infty} \frac{1}{t} E_{x}^{\pi} V\left(x_{N(t)+1}\right)=0 .
$$

Proof:

Step 1. For convenience, we put

$$
w_{x}^{\pi}(t):=E_{x}^{\pi}\left(V\left(x_{N(t)+1}\right) ; N_{C}>N(t)+1\right),
$$

which can be rewritten as

$$
w_{x}^{\pi}(t)=E_{x}^{\pi}\left(\sum_{n=0}^{N_{C}-2} \omega\left(x_{n}, a_{n}, t-T_{n}\right)\right),
$$


with

$$
\omega(x, a, t):=E_{x}^{a}(V(y) ; y \notin C, T>t) .
$$

Here $y$ denotes the next state. Recall that $T$ is the sojourn time in the state $x$.

Step 2. We claim that $w_{x}^{\pi}(t)$ is nonincreasing in $t$. This fact follows immediately from the optional sampling theorem. For this note that

(I) $V\left(x_{n}\right) 1_{\left[N_{C}>n\right]}, n \geq 1$ is supermartingale with respect to $\mathcal{F}_{n}$ :

$$
\begin{aligned}
E_{x}^{\pi}\left(V\left(x_{n+1}\right) 1_{\left[N_{C}>n+1\right]} \mid \mathcal{F}_{n}\right) & \leq E_{x}^{\pi}\left(V\left(x_{n+1}\right) 1_{\left[N_{C} \geq n+1\right]} \mid \mathcal{F}_{n}\right) \\
\leq \lambda V\left(x_{n}\right) 1_{\left[N_{C} \geq n+1\right]} & \leq V\left(x_{n}\right) 1_{\left[N_{C}>n\right]}
\end{aligned}
$$

(II)

$$
w_{x}^{\pi}(t) \leq \sum_{n=1}^{\infty} E_{x}^{\pi}\left(V\left(x_{n}\right) 1_{\left[N_{C}>n\right]}\right) \leq \frac{\lambda V(x)+\eta 1_{C}(x)}{1-\lambda}<\infty
$$

(III)

$$
\lim _{n \rightarrow \infty} E_{x}^{\pi}\left(V\left(x_{n}\right) 1_{\left[N_{C}>n\right]} ; N(t) \geq n\right) \leq \lim _{n \rightarrow \infty} E_{x}^{\pi}\left(V\left(x_{n}\right) 1_{\left[N_{C}>n\right]}\right)=0 .
$$

It is easy to see that (II) and (III) follow from Lemma 1(a),(b).

Applying the optional sampling theorem $[9,14]$ to the above uniformly integrable supermartingale, where $N\left(t_{1}\right)+1 \leq N\left(t_{2}\right)+1\left(t_{1}<t_{2}\right)$ are two stopping stopping times, we get

$$
w_{x}^{\pi}\left(t_{1}\right) \geq w_{x}^{\pi}\left(t_{2}\right)
$$

for all $x \in X$ and $\pi \in \Pi$.

Step 3. Put

$$
w_{x}(t):=\sup _{\pi \in \Pi} w_{x}^{\pi}(t)
$$

for $x \in X, t \geq 0$. By Lemma $4(\mathrm{~b}), w_{x}(t)$ is universally measurable in $x$ for each $t \geq 0$. Note that by $(4)$

$$
w_{x}^{\pi}(t)=E_{x}^{\pi}\left(\sum_{n=0}^{N_{C}-2} \omega\left(x_{n}, a_{n}, t-T_{n}\right)\right)=E_{x}^{\pi}\left(\omega\left(x, a_{0}, t\right)+1_{\left[x_{1} \notin C, T_{1} \leq t\right]} w_{x_{1}}^{\pi\left[x, a_{0}\right]}\left(t-T_{1}\right)\right) .
$$

Denoting $T_{1}$ by $T$ and $x_{1}$ by $y$, we obtain

$$
w_{y}^{\pi\left[x, a_{0}\right]}(t-T) 1_{[y \notin C, T \leq t]}=w_{y}^{\pi\left[x, a_{0}\right]}(t-T) 1_{\left[y \notin C, 0 \leq T \leq \frac{t}{2}\right]}+w_{y}^{\pi\left[x, a_{0}\right]}(t-T) 1_{\left[y \notin C, \frac{t}{2}<T \leq t\right]} .
$$

Taking into account (5) and Lemma 5(b), we observe that

$$
w_{y}^{\pi\left[x, a_{0}\right]}(t-T) \leq w_{y}(t-T) .
$$

This, (7) and the monotonicity of the function $t \mapsto w_{y}(t)$ (Step 2) imply that

$$
\begin{aligned}
w_{y}^{\pi\left[x, a_{0}\right]}(t-T) 1_{[y \notin C, T \leq t]} & \leq w_{y}(t-T) 1_{\left[y \notin C, 0 \leq T \leq \frac{t}{2}\right]}+ \\
w_{y}(t-T) 1_{\left[y \notin C, \frac{t}{2}<T \leq t\right]} & \leq w_{y}\left(\frac{t}{2}\right) 1_{[y \notin C]}+w_{y}(0) 1_{\left[y \notin C, \frac{t}{2}<T\right]} .
\end{aligned}
$$


By Lemma $5(\mathrm{a})$, both functions $w_{y}(0)$ and $w_{y}\left(\frac{t}{2}\right)$ are universally measurable on the state space. Expressions (6) and (8) yield

$$
w_{x}^{\pi}(t) \leq \sup _{a \in A(x)} \omega(x, a, t)+E_{x}^{\pi}\left(w_{y}\left(\frac{t}{2}\right) 1_{[y \notin C]}\right)+E_{x}^{\pi} w_{y}(0) 1_{\left[y \notin C, \frac{t}{2}<T\right]} .
$$

By $(\mathbf{I})$, for $\varepsilon>0$ there exists a constant $S>0$ such that for $t>S$

$$
\sup _{x \in C} \sup _{a \in A(x)} P_{x}^{a}(T>t) \leq \frac{\varepsilon}{3 k\left[\lambda v_{C}+\eta\right]}
$$

where $k$ is an integer satisfying

$$
k>\frac{1}{\ln \lambda} \ln \frac{\varepsilon(1-\lambda)}{3\left[\lambda v_{c}+\eta\right]} .
$$

Let $x \in C$ and $t>2 S$. We obtain the following upper bounds

$$
\sup _{x \in C} \sup _{a \in A(x)} \omega(x, a, t) \leq \frac{\varepsilon}{3 k}
$$

and

$$
E_{x}^{\pi}\left(w_{y}(0) 1_{\left[y \notin C, \frac{t}{2}<T\right]}\right) \leq \sup _{x \in C} \sup _{a \in A(x)}\left(\int_{X} w_{y}(0) 1_{[y \notin C]} q(d y \mid x, a) \int_{\frac{t}{2}}^{\infty} P_{x}^{a}\left(d t^{\prime}\right)\right) \leq \frac{\varepsilon}{3 k} .
$$

From (9), (10), and (11), we get

$$
w_{x}^{\pi}(t) \leq 2 \frac{\varepsilon}{3 k}+\sup _{a \in A(x)} \int_{X} w_{y}\left(\frac{t}{2}\right) 1_{[y \notin C]} q(d y \mid x, a) .
$$

Put

$$
\gamma(y):=w_{y}\left(\frac{t}{2}\right) 1_{[y \notin C]},
$$

for $y \in X$. Then $\|\gamma\|_{V}<\infty$ and $\gamma$ is universally measurable. By Lemma 8.3.7(a) [5] and F 3.9 [15], the function

$$
a \mapsto \int_{X} \gamma(y) q(d y \mid x, a)
$$

is continuous on $A(x)$. Note also, that

$$
x \mapsto \int_{X} \gamma(y) q(d y \mid x, a)
$$

is universally measurable, Proposition 7.46 [1] or F 3.8 [15]. Hence, by F 2.7 [15], there exists a universally measurable control function $u$ such that

$$
\max _{a \in A(x)} \int_{X} \gamma(y) q(d y \mid x, a)=\int_{X} \gamma(y) q(d y \mid x, u)
$$


for each $x \in X$. By (12) and (13), we have

$$
w_{x}(t)=\sup _{\pi \in \Pi} w_{x}^{\pi}(t) \leq 2 \frac{\varepsilon}{3 k}+\int_{X} w_{y}\left(\frac{t}{2}\right) 1_{[y \notin C]} q(d y \mid x, u) .
$$

Iteration of the last inequality $(k-1)$ times together with Step 2(II) and Lemma 1(a) (which is also valid for universally measurable policies) gives

$$
\begin{array}{r}
\operatorname{supsup}_{x \in C} w_{x \in \Pi}^{\pi}(t) \leq \frac{2 \varepsilon}{3}+\sup _{x \in C} E_{x}^{u}\left(w_{x_{k}}\left(\frac{t}{2^{k}}\right) ; N_{C}>k\right) \\
\leq \frac{2 \varepsilon}{3}+\sup _{x \in C} E_{x}^{u}\left(\frac{\lambda}{1-\lambda} V\left(x_{k}\right) ; N_{C}>k\right) \leq \varepsilon .
\end{array}
$$

Step 4. Set

$$
z_{x}^{\pi}(t):=E_{x}^{\pi}\left(V\left(x_{N(t)+1}\right) ; N_{C} \geq N(t)+1\right)
$$

and

$$
z(t):=\sup _{x \in C} \sup _{a \in A(x)} z_{x}^{\pi}(t)
$$

Proceeding analogously as in Step 2, we note that $z(t)$ is a nonincreasing function in $t$ and $z(t)<\infty$. Moreover, using the strong Markov property and Lemma $5(\mathrm{~b})$, we obtain

$$
\begin{aligned}
& E_{x}^{\pi} V\left(x_{N(t)+1}\right)=z_{x}^{\pi}(t)+ \\
& \quad \sum_{m=1}^{\infty} E_{x}^{\pi}\left(E_{x}^{\pi}\left[V\left(x_{N(t)+1}\right) ; N_{C}(m+1) \geq N(t)+1>N_{C}(m) \mid \mathcal{F}_{N_{C}(m)}\right]\right) \\
& \quad \leq z_{x}^{\pi}(t)+ \\
& \quad \sum_{m=1}^{\infty} E_{x}^{\pi}\left(E_{x_{m}}^{\pi\left[h_{m}^{\prime}\right]}\left[V\left(x_{N\left(t-T_{m}\right)+1}\right) ; N_{C} \geq N\left(t-T_{m}\right)+1\right] ; x_{m} \in C, T_{m} \leq t\right) \\
& \quad \leq z_{x}^{\pi}(t)+ \\
& \quad \sum_{m=1}^{\infty} E_{x}^{\pi}\left(\sup _{\bar{x} \in C \hat{\pi} \in \Pi} E_{\bar{x}}^{\hat{\pi}}\left[V\left(x_{N\left(t-T_{m}\right)+1}\right) ; N_{C} \geq N\left(t-T_{m}\right)+1\right] ; x_{m} \in C, T_{m} \leq t\right) \\
& \quad=z_{x}^{\pi}(t)+\int_{0}^{t} z(t-u) M_{x}^{\pi}(d u) .
\end{aligned}
$$

Step 5. Now it remains only to prove that $\lim _{t \rightarrow \infty} z(t)=0$. This follows from

$$
z(t) \leq \sup _{x \in C} \sup _{\pi \in \Pi} w_{x}^{\pi}(t)+\sup _{x \in C} \sup _{\pi \in \Pi} E_{x}^{\pi}\left(V\left(x_{N(t)+1}\right) ; N_{C}=N(t)+1\right)
$$

and

$$
\begin{aligned}
& \sup _{x \in C} \sup _{\pi \in \Pi} E_{x}^{\pi}\left(V\left(x_{N_{C}}\right) ; N_{C}=N(t)+1\right) \leq v_{C} \sup _{x \in C} \sup _{\pi \in \Pi} P_{x}^{\pi}\left(T_{N_{C}}>t\right) \\
& \leq v_{C} \sup _{x \in C} \sup _{\pi \in \Pi} \frac{E_{x}^{\pi}\left(T_{N_{C}}\right)}{t} \leq \frac{B \phi_{C}}{t} .
\end{aligned}
$$


The sequence of inequalities is due to assumption (GE,ii), Markov inequality and Lemma 1(b) (see also (B, iv)), respectively. Hence, by (14) and the last expression $z(t) \rightarrow 0$ as $t \rightarrow \infty$.

The desired assertion is a consequence of

$$
E_{x}^{\pi} V\left(x_{N(t)+1}\right) \leq z_{x}^{\pi}(t)+\int_{0}^{t} z(t-u) M_{x}^{\pi}(d u) \quad(\text { by Step } 4),
$$

Lemma $4(\mathrm{c})$ and the fact that

$$
z_{x}^{\pi}(t) \leq \frac{\lambda+\eta}{1-\lambda} V(x)
$$

which follows from Lemma 1(a).

Lemma 8: For any $\pi \in \Pi, x \in X$, we have:

$$
\lim _{t \rightarrow \infty} \frac{1}{t} E_{x}^{\pi} T_{N(t)+1}=1
$$

Proof: Note that

$$
\frac{t}{t} \leq E_{x}^{\pi} \frac{T_{N(t)+1}}{t} \leq \frac{E_{x}^{\pi} T_{N(t)}+E_{x}^{\pi}\left(T_{N(t)+1}-T_{N(t)}\right)}{t} \leq \frac{t+B}{t}
$$

where $B$ is from assumption $(\mathbf{B}$, iv). The last inequality is due to the fact that $[N(t)+1=$ $n] \in \mathcal{F}_{n}$ and

$$
\begin{aligned}
E_{x}^{\pi}\left(T_{N(t)+1}-T_{N(t)}\right) & =\sum_{n=1}^{\infty} E_{x}^{\pi}\left(T_{n+1}-T_{n}\right) 1_{[N(t)+1=n]} \\
& =\sum_{n=1}^{\infty} E_{x}^{\pi}\left(E_{x}^{\pi}\left[\left(T_{n+1}-T_{n}\right) 1_{[N(t)+1=n]} \mid \mathcal{F}_{n}\right]\right) \\
& =\sum_{n=1}^{\infty} E_{x}^{\pi}\left(\tau\left(x_{n}, a_{n}\right) 1_{[N(t)+1=n]}\right) \leq B .
\end{aligned}
$$

Proof of Theorem: (a) We claim that

$$
S_{n}:=\sum_{k=0}^{n-1}\left(c\left(x_{k}, a_{k}\right)-g \tau\left(x_{k}, a_{k}\right)\right)+h\left(x_{n}\right)
$$

is a submartingale with respect to $\mathcal{F}_{n}$. This follows from the optimality equation (3), because

$$
h(x) \leq c(x, a)-g \tau(x, a)+\int h(y) q(d y \mid x, a) .
$$


In order to apply optional sampling theorem for this submartingale, where $N(t)+1$ is a stopping time, we have to check that

(I) $E_{x}^{\pi}\left|S_{N(t)+1}\right|$ is well defined;

(II) $E_{x}^{\pi}\left[\left|S_{n}\right| ; N(t) \geq n\right]$ tends to zero, when $n \rightarrow \infty$.

For (I), it holds

$$
E_{x}^{\pi}\left|S_{N(t)+1}\right| \leq E_{x}^{\pi}\left(\sum_{k=0}^{N(t)}\left|c\left(x_{k}, a_{k}\right)\right|\right)+g E_{x}^{\pi}\left(\sum_{k=0}^{N(t)} \tau\left(x_{k}, a_{k}\right)\right)+\|h\|_{V} E_{x}^{\pi} V\left(x_{N(t)+1}\right) .
$$

These expressions are finite by Lemmas 6 and $7\left(h \in L_{V}^{\infty}\right)$. Furthermore,

$$
\begin{aligned}
& E_{x}^{\pi}\left(\left|S_{n}\right| ; N(t) \geq n\right) \leq \\
& \quad E_{x}^{\pi}\left(\sum_{k=0}^{N(t)}\left(\left|c\left(x_{k}, a_{k}\right)\right|+g \tau\left(x_{k}, a_{k}\right) ; N(t) \geq n\right)\right)+\|h\|_{V} E_{x}^{\pi}\left(V\left(x_{n}\right) ; N(t) \geq n\right) .
\end{aligned}
$$

Taking into account Remark 2, these terms go to zero by Lemma 6 (the first one) and by Lemma 1(b) (the second one). Finally, we obtain

$$
h(x) \leq E_{x}^{\pi}\left(\sum_{k=0}^{N(t)}\left(c\left(x_{k}, a_{k}\right)-g \tau\left(x_{k}, a_{k}\right)\right)\right)+E_{x}^{\pi} h\left(x_{N(t)+1}\right)
$$

and consequently,

$$
g \frac{1}{t} E_{x}^{\pi} T_{N(t)+1} \leq \frac{1}{t} E_{x}^{\pi}\left(\sum_{k=0}^{N(t)} c\left(x_{k}, a_{k}\right)\right)+E_{x}^{\pi}\left(\frac{h\left(x_{N(t)+1}\right)}{t}\right)-\frac{h(x)}{t} .
$$

The left side tends to $g$ (Lemma 8), whilst the right side goes to $j(x, \pi)$, defined in $(2)$ (Lemma 7).

(b) Let $f \in F$ be fixed. Then there exists a function $h_{f} \in L_{V}^{\infty}$ for which Poisson's equation holds, i.e.

$$
h_{f}(x)=c(x, f)+\int_{X} h_{f}(y) q(d y \mid x, f)-J(x, f) \tau(x, f) .
$$

The arguments used above in particular imply that

$$
S_{n}^{\prime}:=\sum_{k=0}^{n-1}\left(c\left(x_{k}, f\right)-J(x, f) \tau\left(x_{k}, f\right)\right)+h_{f}\left(x_{n}\right)
$$

is a uniformly integrable martingale. (We recall that by our assumption (GE), the ratioaverage cost is independent of the initial state for each stationary policy [5].) Applying Doob's theorem, we get

$$
h_{f}(x)=E_{x}^{f}\left(\sum_{k=0}^{N(t)}\left(c\left(x_{k}, f\right)-J(x, f) \tau\left(x_{k}, f\right)\right)\right)+E_{x}^{f} h_{f}\left(x_{N(t)+1}\right) .
$$


This gives the result.

REMARK 3: In the proof it is assumed that $\tau(x, a)<B(\mathbf{B}$, iv). However, the optimality equation (3) remains true, if we allow for unbounded mean holding time, i.e.

$$
\tau(x, a)<B_{1} V(x)
$$

for some constant $B_{1}$. The direct proof is provided in [24]. The reader can also follow the proof given in [7] with slight modification of the constants in Theorem 1 . Then, the minor corrections in the proof of Lemmas 5 (b) and 7 give the equivalence of expected average costs, (1) and (2), for stationary policies.

REMARK 4: Our main theorem has some relevance to studying stochastic games with Borel state space. Namely, the results given in $[8,16]$ for semi-Markov games remains also valid for time-average criterion (2).

Acknowledgment: I wish to thank Prof. Eugene Feinberg and Prof. Andrzej S. Nowak for suggesting the problem, many helpful discussions and strong encouragment.

\section{References}

[1] D.P. Bertsekas And S.E. Shreve, Stochastic Optimal Control: The Discrete Time Case, Academic Press, New York, 1978.

[2] D. Blackwell, A renewal theorem, Duke Math. J., 15 (1948), pp. 145-150.

[3] L.D. Brown and R. Purves, Measurable selections of extrema, Ann. Stat., 1 (1973), pp. 902-912.

[4] E.A. Feinberg, Constrained semi-Markov decision processes with average rewards, Math. Methods Oper. Res., 39 (1994), pp. 257-288.

[5] O. Hernández-Lerma and J.B. Lasserre, Further Topics on Discrete-Time Markov Control Process, Springer-Verlag, New York, 1999.

[6] O. Hernández-Lerma and F. Luque-VÁsquez, Semi-Markov control models with average costs, Applicationes Mathematicae, 26 (1999), pp. 315-331.

[7] A. JAŚKIEWICZ, An approximation approach to ergodic semi-Markov control processes, Math. Methods Oper. Res., 54 (2001), pp. 1-19.

[8] A. Jaśkiewicz, Zero-sum semi-Markov games, SIAM J. Control Optim., 41 (2002), pp. $723-739$.

[9] A.P. Maitra And W.D.Sudderth, Discrete Gambling and Stochastic Games, SpringerVerlag, New York,1996. 
[10] S.P. Meyn And R.L. Tweedie, Markov Chains and Stochastic Stability, Springer-Verlag, New York, 1993.

[11] S.P. Meyn And R.L. Tweedie, Computable bounds for geometric convergence rates of Markov chains, Ann. Appl. Probab., 4 (1994), pp. 981-1011.

[12] H. Mine and S. Osaki, Markovian Decision Processes, Elsevier, New York, 1970.

[13] J. Neveu, Mathematical Foundations of the Calculus of Probability, Holden-Day, San Francisco, 1965.

[14] J. Neveu, Discrete-Parameter Martingales, Elsevier, New York, 1975.

[15] A.S. NowAK, Universally measurable strategies in zero-sum stochastic games, Ann. Probab., 13 (1985), pp. 269-287.

[16] A.S. NowAK, Some remarks on equilibria in semi-Markov games, Applicationes Mathematicae, 27 (2000), pp. 385-394.

[17] A.S. Nowak And E. Altman E-Equilibria for stochastic games with uncountable state space and unbounded costs, SIAM J. Control Optim., 40 (2002), pp. 1821-1839.

[18] L.M. Puterman, Markov Decision Processes, John Wiley, New York, 1994.

[19] S.M. Ross, Applied Probability Models with Optimization Applications, Holden-Day, San Francisco, 1970.

[20] M. ScHÄL, On the second optimality equation for semi-Markov decision models, Math. Oper. Res., 17 (1992), pp. 470-486.

[21] L.I. Sennott, Average cost semi-Markov decision processes and the control of queueing system, Probability in the Engineering and Informational Sciences, 3 (1989), pp. 247-272.

[22] R.E. Strauch, Negative dynamic programming, Ann. Stat., 37 (1966), pp. 871-890.

[23] A. Yushkevich, On semi-Markov controlled models with an average reward criterion, Theory Probab. Appl., 26 (1981), pp. 796-803.

[24] O. Vega-Amaya And F. Luque-VÁsquez, Sample-path average cost optimality for semiMarkov control processes on Borel spaces: unbounded costs and mean holding times, Applicationes Mathematicae, 27 (2000), pp. 343-367. 
Corrigendum to "On the equivalence of two expected average cost criteria for semiMarkov control processes" published in Math. Oper. Res. 29(2), 326-338, 2004.

\section{Anna Jaśkiewicz}

Institute of Mathematics and Computer Science, Wrocław University of Technology, Wybrzeże Wyspiańskiego 27, 50-370 Wrocław, Poland (e-mail: anna.jaskiewicz@pwr.wroc.pl)

Lemma 8 in [1] is incorrect. Its proof requires additional assumption. Uniform integrability condition (I) on p. 329 should be formulated as follows.

I Uniform integrability condition:

(i) $\lim _{t \rightarrow \infty} \sup _{x \in C} \sup _{a \in A(x)} P_{x}^{a}(T>t)=0$.

(ii) There exists a decreasing function $\kappa$ such that $\kappa(0) \leq B, \kappa(+\infty)=0$ and

$$
E_{x}^{a} T 1_{[T>t]} \leq \kappa(t)
$$

for all $(x, a) \in K . B$ is a constant from assumption (B, iv).

Similar assumption to (I, ii) has been also used in $[2,3]$.

Lemma 8: For any $\pi \in \Pi, x \in X$, we have:

$$
\lim _{t \rightarrow \infty} \frac{1}{t} E_{x}^{\pi} T_{N(t)+1}=1
$$

Proof: Note that

$$
\frac{t}{t} \leq E_{x}^{\pi} \frac{T_{N(t)+1}}{t} \leq \frac{E_{x}^{\pi} T_{N(t)}+E_{x}^{\pi}\left(T_{N(t)+1}-T_{N(t)}\right)}{t} \leq 1+\frac{E_{x}^{\pi}\left(T_{N(t)+1}-T_{N(t)}\right)}{t} .
$$

We show that the last term on the right-hand side in the above display tends to 0 . Indeed, observe that

$$
\begin{aligned}
& E_{x}^{\pi}\left(T_{N(t)+1}-T_{N(t)}\right)=\sum_{n=0}^{\infty} E_{x}^{\pi}\left(T_{n+1}-T_{n}\right) 1_{[N(t)=n]} \\
& =\sum_{n=0}^{\infty} E_{x}^{\pi}\left(E_{x}^{\pi}\left[\left(T_{n+1}-T_{n}\right) 1_{\left[T_{n} \leq t<T_{n+1}\right]} \mid \mathcal{F}_{n}\right]\right) \\
& =\sum_{n=0}^{\infty} E_{x}^{\pi}\left(E_{x}^{\pi}\left[\left(T_{n+1}-T_{n}\right) 1_{\left[t-T_{n}<T_{n+1}-T_{n}\right]} \mid \mathcal{F}_{n}\right] 1_{\left[0 \leq t-T_{n}\right]}\right) \\
& =\sum_{n=0}^{\infty} E_{x}^{\pi}\left(E_{x_{n}}^{\pi\left[h_{n}\right]}\left[T 1_{\left[t-T_{n}<T\right]}\right] 1_{\left[0 \leq t-T_{n}\right]}\right) \\
& \leq \sum_{n=0}^{\infty} E_{x}^{\pi}\left[\kappa\left(t-T_{n}\right) 1_{\left[T_{n} \leq t\right]}\right]=E_{x}^{\pi}\left[\sum_{n=0}^{N(t)} \kappa\left(t-T_{n}\right)\right] \\
& =E_{x}^{\pi}\left(\sum_{n=0}^{N(t-\sqrt{t})} \kappa\left(t-T_{n}\right)+1_{[N(t-\sqrt{t})+1 \leq N(t)]} \sum_{n=N(t-\sqrt{t})+1}^{N(t)} \kappa\left(t-T_{n}\right)\right)
\end{aligned}
$$




$$
\begin{aligned}
& \leq E_{x}^{\pi}\left(\sum_{n=0}^{N(t-\sqrt{t})} \kappa(\sqrt{t})+1_{[N(t-\sqrt{t})+1 \leq N(t)]} \sum_{n=N(t-\sqrt{t})+1}^{N(t)} \kappa(0)\right) \\
& \leq \kappa(\sqrt{t}) E_{x}^{\pi}(N(t-\sqrt{t})+1)+B E_{x}^{\pi}(N(t)-N(t-\sqrt{t}))
\end{aligned}
$$

From Lemma 6(b) in [1] it follows that

$$
\kappa(\sqrt{t}) E_{x}^{\pi}(N(t-\sqrt{t})+1) \leq \kappa(\sqrt{t})\left(\phi(x)+\phi_{C} M(t)+1\right),
$$

where $\phi$ is defined in Lemma 3(b) in [1]. Furthermore, from the proof of Lemma 4(c) in [1], we get that

$$
\frac{\kappa(\sqrt{t})\left(\phi(x)+\phi_{C} M(t)+1\right)}{t} \rightarrow 0 \quad \text { as } \quad t \rightarrow \infty .
$$

Let us now consider the second term. Observe that by the strong Markov property it follows that

$$
\begin{aligned}
& E_{x}^{\pi}(N(t)-N(t-\sqrt{t})-1+1)=1+E_{x}^{\pi}\left[E_{x}^{\pi}\left(N(t)-N(t-\sqrt{t})-1 \mid \mathcal{F}_{N(t-\sqrt{t})+1}\right)\right] \\
& =1+E_{x}^{\pi}\left[E_{\left.x_{N(t-\sqrt{t})+1}^{\pi\left[h_{N(-\sqrt{t})+1}\right]}(N(t)-N(t-\sqrt{t})-1)\right]}\right. \\
& \leq 1+E_{x}^{\pi}\left[E_{\left.x_{N(t-\sqrt{t})+1}^{\pi\left[h_{N(t-\sqrt{t})+1}\right]} N(t-t+\sqrt{t})\right]}\right. \\
& \leq 1+E_{x}^{\pi}\left[\phi\left(x_{N(t-\sqrt{t})+1}\right)+\phi_{C} M(\sqrt{t})\right] \text {, }
\end{aligned}
$$

where the second inequality is due to Lemma 6(b) in [1] (put $\tau \equiv 1$ ). Since $v \geq 1$, we have that

$$
E_{x}^{\pi} \phi\left(x_{N(t-\sqrt{t})+1}\right) \leq C_{1} E_{x}^{\pi} V\left(x_{N(t-\sqrt{t})+1}\right)
$$

with $\left.C_{1}:=-1 / \ln \lambda(1+\eta / \lambda)\right)$. Now by Lemma 7 in [1] and the argument used in the proof of Lemma $4(\mathrm{c})$ in [1], it follows that

$$
0 \leq \frac{E_{x}^{\pi}(N(t)-N(t-\sqrt{t}))}{t} \leq \frac{1}{t}+C_{1} \frac{E_{x}^{\pi} V\left(x_{N(t-\sqrt{t})+1}\right)}{t-\sqrt{t}} \frac{t-\sqrt{t}}{t}+\phi_{C} \frac{M(\sqrt{t})}{\sqrt{t}} \frac{1}{\sqrt{t}} \rightarrow 0 .
$$

This proves the assertion.

\section{References}

[1] A. JAśKiewicz, On the equivalence of two expected average cost criteria for semi-Markov control processes, Math. Oper. Res. 29 (2004) pp. 326-338.

[2] M. ScH ̈̈L, On the second optimality equation for semi-Markov decision models, Math. Oper. Res., 17 (1992), pp. $470-486$.

[3] A. Yushkevich, On semi-Markov controlled models with an average reward criterion, Theory Probab. Appl., 26 (1981), pp. 796-803. 